\title{
High-Dose Thoracic Re-irradiation of Lung Cancer Using Highly Conformal Radiotherapy Is Effective with Acceptable Toxicity
}

\author{
Ji Hyun Hong, MD' \\ Yeon-Sil Kim, MD, PhD' \\ Sea-Won Lee, MD \\ So Jung Lee, $M D^{1}$ \\ Jin Hyung Kang, MD, PhD² \\ Suk Hee Hong, MD, PhD2 \\ Ju-Young Hong, MS \\ GeumSeong Cheon, $\mathrm{MS}^{1}$
}

\begin{abstract}
Purpose
Thoracic re-irradiation (re-RT) of lung cancer has been challenged by the tolerance doses of normal tissues. We retrospectively analyzed local control, overall survival (OS) and toxicity after thoracic re-RT using highly conformal radiotherapy, such as intensity modulated radiotherapy and stereotactic body radiotherapy.
\end{abstract}

\section{Materials and Methods}

Thirty-one patients who received high-dose thoracic re-RT were analyzed. Doses were recalculated to determine biologically equivalent doses. The median interval to re-RT was 15.1 months (range, 4.4 to 56.3 months), the median initial dose was $79.2 \mathrm{~Gy}_{10}$ (range, 51.75 to $150 \mathrm{~Gy}_{10}$ ), and the median re-RT dose was $68.8 \mathrm{~Gy}_{10}$ (range, 43.2 to $132 \mathrm{~Gy}_{10}$ ).

\section{Results}

Eighteen (58.1\%) and eleven (35.5\%) patients showed loco-regional recurrence and distant metastasis, respectively, after 17.4 months of median follow-up. The 1-year and 2-year local control rates were $60.2 \%$ and $43.7 \%$, respectively. The median loco-regional recurrencefree-survival (LRFS) was 15.4 months, and the median OS was 20.4 months. The cumulative and re-RT biologically equivalent dose for $\alpha / \beta=10\left(B E D_{10}\right)$ doses were the most significant prognostic factors. Cumulative $\mathrm{BED}_{10} \geq 145 \mathrm{~Gy}_{10}$ and re-RT $\mathrm{BED}_{10} \geq 68.7 \mathrm{~Gy}_{10}$ were significantly associated with longer OS ( $p=0.029$ and $p=0.012$, respectively) and LRFS ( $p=0.003$ and $p=0.000$, respectively). The most frequent acute toxicity was grade $1-2$ pulmonary toxicity (41.9\%). No acute grade 3 or higher toxicities occurred.

\section{Conclusion}

Our results show that high-dose thoracic re-RT of lung cancer can be safely delivered using highly conformal radiotherapy with favorable survival and acceptable toxicity. An optimal strategy to select patients who would benefit from re-RT is crucial in extending the indications and improving the efficacy with a sufficiently high dose.

\author{
Correspondence: Yeon-Sil Kim, MD, PhD \\ Department of Radiation Oncology, \\ Seoul St. Mary's Hospital, College of Medicine, \\ The Catholic University of Korea, \\ 222 Banpo-daero, Seocho-gu, Seoul 06591, Korea \\ Tel: 82-2-2258-6259 \\ Fax: 82-2-2258-1532 \\ E-mail: yeonkim7@catholic.ac.kr \\ Received August 21, 2018 \\ Accepted November 28, 2018 \\ Published Online November 29, 2018
}

\section{Key words}

Lung neoplasms, Re-irradiation, Intensity modulated radiotherapy, Radiosurgery

\section{Introduction}

Lung cancer is the second most common cancer in the world and the fourth most common cancer in Korea. In Korea, 23,177 patients were newly diagnosed in 2013 and 17,330 lung cancer patients died in 2014 [1]. Lung cancer has been the leading cause of cancer death in Korea since 1983. The prevalence of lung cancer is still increasing and expected to continue increasing for the next 10 years [2].

In lung cancer, radiotherapy (RT) plays a major role in treatment with curative intent $[3,4]$. However, the 5-year loco-regional progression rate of non-small cell lung cancer (NSCLC) with initial concurrent RT treatment is 28.9\% [5], and local failure of small cell lung cancer (SCLC) treatment occurs in up to $36 \%-52 \%$ of patients with initial concurrent RT [6]. Locoregional disease recurrence continues to be a major problem and has been the main cause of death after 
initial definitive chemoradiotherapy or RT.

Since there are few prospective trials for high-dose re-irradiation (re-RT) in the thorax, the effect and safety of high dose re-RT is uncertain. High-dose re-RT might cause severe radiation injury, such as radiation pneumonitis, esophagitis, fistula, and stenosis. Furthermore, the tolerance of normal tissues in the context of re-RT is unknown. However, owing to recent technological advances in RT and imaging techniques, high-dose re-RT is now considered to be a potential salvage treatment approach [7-9].

In this study, we retrospectively analyzed our institutional experience to evaluate cancer control parameters, including local control (LC), loco-regional progression-free survival (LRFS), local progression-free survival (LPFS), and overall survival (OS), as well as the toxicities of re-RT using highly conformal RT, such as intensity modulation radiotherapy (IMRT) or stereotactic body radiotherapy (SBRT).

\section{Materials and Methods}

\section{Patients}

From August 2005 to November 2016, 56 patients with lung cancer received a second course of thoracic RT. Each patient was discussed in a multidisciplinary team with thoracic surgeons, respiratory physicians, radiologists and medical oncologist before they decided to receive a second course of RT. Patients who satisfied the following criteria were included in the study: (1) the primary tumor was histologically diagnosed as NSCLC or SCLC; (2) recurrences were confirmed by documented radiographic findings and/or pathological biopsies within the thoracic area; (3) patients had received re-RT to the thorax; (4) patients had received curative intent RT of more than 50 Gy for NSCLC and for patients with SCLC who were treated with radical concurrent chemoradiation (CCRT), with 45 Gy twice a day; and (5) there were no active distant metastasis or controlled solitary distant metastasis at the time of re-RT.

Patient charts were reviewed, and the following data were collected: sex, smoking history, surgery history, history of chemotherapy, Eastern Cooperative Oncology Group (ECOG) performance status (PS) before re-RT, tumor histology, stages at initial RT and second RT, planning tumor volume (PTV), and gross tumor volume (GTV) at the first and second RT $(\mathrm{mL}), \mathrm{RT}$ doses, biologically equivalent dose (BED, $\alpha / \beta=10$ and 3), fraction size of at the first and second RT, RT techniques of the initial course and of re-RT, time interval between RT courses, acute and chronic toxicities, survival duration, LC, LRFS, LPFS, and distant metastasis-free survival
(DMFS). All of the tumors were staged using the 7th edition of the American Joint Committee on Cancer (AJCC) staging.

\section{Treatment/radiotherapy}

For treatment planning, pretreatment imaging was obtained in the supine position. For the re-RT, 4-dimensional computed tomography (4D-CT) was taken routinely. The primary tumor and enlarged lymph nodes were contoured as the GTV on axial treatment planning CT based on the pretreatment chest CT and/or fluorine-18-2-fluoro-2-deoxyD-glucose positron emission tomography and computed tomography (FDG-PET/CT). The clinical target volume (CTV) was defined as a $0.5 \mathrm{~cm}$ expansion from the GTV, and the PTV was determined by expanding the CTV by adequate margins from the tumor movement assessed in 4D-CT.

The treatments were delivered using the three-dimensional conformal radiotherapy (3D-CRT) technique with a linear accelerator for the initial RT, except for four patients who were treated using IMRT technique. For re-RT, every treatment was administered using daily volumetric imageguidance RT using kilovoltage or megavoltage cone beam CT from a highly conformal treatment modality, such as IMRT or helical tomotherapy (TOMO). A noncoplanar field technique was used for IMRT or SBRT. When patients have lesions close proximity to trachea or esophagus, to spare the region of interests more efficiently, TOMO were used for a re-RT plan.

\section{Follow-up and toxicity analysis}

After the re-RT was completed, patients were regularly followed up at 2-3 month intervals with examinations, such as blood tests, chest X-rays, chest computed tomography (CT), and FDG-PET/CT if needed. LC was defined as no progressive disease in the high-dose treatment volume within $50 \%$ of the isodose line.

The OS time after re-RT was the time between the first day of the second RT and the date of the patient's death or most recent follow-up visit. LPFS was defined as the time between the first day of the second RT and date of recurrence in the re-irradiated high-dose treatment volume. LRFS and DMFS were defined as the time between the first day of the second RT and the date that the loco-regional recurrence or distant metastasis was documented and imaged.

The severity of the acute and late toxicities was evaluated by the Common Terminology Criteria for Adverse Events (CTCAE) ver. 4.03. Acute toxicities were defined as adverse effects that appeared within 3 months from the start of RT. The adverse effects that appeared after three months were defined as late toxicities. 
Table 1. Characteristic of patients and tumors

\begin{tabular}{|cc}
\hline Characteristic & Value \\
Age at re-RT (yr) & $63.6(43.6-88.9)$ \\
Sex & $27(87.1)$ \\
Male & $4(12.9)$ \\
\hline Female & $25(80.6)$ \\
\hline History of tobacco use & $9(29.0)$ \\
\hline History of thoracic surgery & \\
\hline ECOG PS at re-RT & $6(19.4)$ \\
\hline 0 & $23(74.2)$ \\
\hline 1 & $2(6.5)$ \\
\hline 2 & \\
\hline Histology & $15(48.4)$ \\
\hline NSCLC & $8(25.8)$ \\
\hline SqCC & $1(3.2)$ \\
\hline Adenoca & $7(22.6)$ \\
\hline Others, large cell & \\
\hline SCLC & \\
\hline Initial AJCC stage & $3(9.7)$ \\
\hline NSCLC & $4(13)$ \\
\hline I & $14(45.1)$ \\
\hline II & $2(6.4)$ \\
\hline III & $1(3.2)$ \\
\hline IV & $5(16.1)$ \\
\hline Unknown & $2(6.5)$ \\
\hline LD & \\
\hline ED & \\
\hline
\end{tabular}

Values are presented as median (range) or number (\%). re-RT, re-irradiation; ECOG PS, Eastern Cooperative Oncology Group performance status; NSCLC, non-small cell lung cancer; SqCC, squamous cell carcinoma; Adenoca, adenocarcinoma; SCLC, small cell lung cancer; AJCC stage, American Joint Committee on Cancer seventh edition; $\mathrm{LD}$, limited disease; $\mathrm{ED}$, extensive disease.

\section{Statistical analysis}

Survival outcomes were estimated by Kaplan-Meier analysis. Mann-Whitney analysis was used to evaluate the relationship between the RT parameters and toxicities. Cox regression was used to assess the associations between the survival outcomes and predictive factors. A p-value of $<0.05$ was considered significant. Multivariable analysis was precluded by the small number of events. All statistical analyses were performed with SPSS software ver. 23.0 (IBM Corp., Armonk, NY).
Table 2. Overview of the initial RT

\begin{tabular}{lc} 
Parameter & Value \\
\hline Dose (Gy) & $60(45-66)$ \\
NSCLC & $64.5(50-66)$ \\
SCLC & 45 \\
Dose, BED Gy10 & $79.2(51.8-150)$ \\
NSCLC & $79.2(60-150)$ \\
\hline SCLC & 51.8 \\
Fraction size & $2(1.5-15)$ \\
Type of radiation & $27(87.1)$ \\
Conformal & $4(12.9)$ \\
IMRT & \\
Concurrent chemotherapy & $19(61.3)$ \\
Yes & $12(38.7)$ \\
\hline No & \\
RT purpose & $21(67.7)$ \\
Radical & $5(16.1)$ \\
Salvage & $5(16.1)$ \\
Adjuvant & $353.2(33.3-679.9)$ \\
PTV (mL) & $57.4(2.8-211.5)$ \\
\hline GTV (mL)
\end{tabular}

Values are presented as median (range) or number (\%). RT, irradiation; NSCLC, non-small cell lung cancer; SCLC, small cell lung cancer; $\mathrm{BED}_{10}$, biologically equivalent dose for $\alpha / \beta=10$; IMRT, intensity modulation radiotherapy; PTV, planning tumor volume; GTV, gross tumor volume.

\section{Ethical statement}

This study was approved by the Institutional Review Board (IRB) of Seoul St. Mary's Hospital (IRB number: KC17RESI0660). The necessity of informed consent for this retrospective analysis was waived.

\section{Results}

\section{Patient and tumor characteristics}

Thirty-one patients, among which 24 (77.4\%) had NSCLC and seven $(22.6 \%)$ had SCLC, were included in the second course of thoracic re-RT. The patient characteristics are shown in Table 1. The median age at the re-RT was 63.6 years (range, 43.6 to 88.9 years). There were 27 males $(87.1 \%$ ) and four females (12.9\%). Twenty-nine patients $(93.5 \%)$ showed an ECOG PS 0 or 1 at the time of re-RT. Only two patients showed an ECOG 2.

In nine cases $(29 \%)$, pathological confirmation of recur- 
Table 3. Overview of re-RT

\begin{tabular}{|cc}
\hline Characteristic & Value \\
\hline Interval to re-RT & $15.1(4.4-56.3)$ \\
\hline Dose (Gy) & $50(35-65)$ \\
\hline NSCLC & $51.4(45-65)$ \\
SCLC & $45(35-55)$ \\
\hline Dose, BED Gy10 & $68.8(43.2-132)$ \\
\hline NSCLC & $68.8(55.1-132)$ \\
\hline SCLC & $58.5(43.2-70.2)$ \\
\hline Fraction size (Gy) & \\
\hline$\leq 3$ & $21(67.7)$ \\
\hline$>3$ & $10(32.2)$ \\
\hline Re-RT field according to initial RT field & \\
(70\% dose line level) & \\
\hline In-field & $23(74.2)$ \\
\hline Out-field & $8(25.8)$ \\
\hline Laterality according to initial RT field & \\
\hline Ipsilateral & $25(80.6)$ \\
Contralateral & $6(19.4)$ \\
\hline Type of radiation & \\
\hline SBRT & $10(32.3)$ \\
\hline IMRT, TOMO & $21(67.7)$ \\
\hline Concurrent chemotherapy & $3(9.7)$ \\
\hline Yes & $28(90.3)$ \\
\hline No & $51.3(13-299.3)$ \\
\hline Median PTV (mL) & $13.5(1.4-124.9)$ \\
\hline
\end{tabular}

Values are presented as median (range) or number $(\%)$. Interval: the first day of re-RT-the last day of initial RT. re-RT, re-irradiation; NSCLC, non-small cell lung cancer; SCLC, small cell lung cancer; $B E D_{10}$, biologically equivalent dose for $\alpha / \beta=10$; SBRT, stereotactic body radiotherapy; IMRT, intensity modulation radiotherapy; TOMO, tomotherapy; PTV, planning tumor volume; GTV, gross tumor volume.

rence or progression was performed before re-RT. One patient with squamous cell carcinoma at the initial treatment was confirmed as having a non-specified malignancy, and one patient with SCLC at the initial diagnosis was shown to have squamous cell carcinoma with neuroendocrine differentiation. Except for these two patients, the pathologic results were the same as the initial results. Three patients were given CCRT as re-RT because PTV was small as they recurred on a single lymph node and they weren't heavily treated with chemotherapy. Four SCLC patients were treated with chemotherapy after re-RT as courses of treatment. For NSCLC patients, ten patients hadn't received treatment including radiation, surgery, and chemotherapy until progressions of disease had found. However, after the progressions have found, chemotherapy was given. Five NSCLC patients were given chemotherapy after re-RT as courses of the treatment because their recurred tumors burden was large with the nodal chain metastasis or large size of the recurrent tumor. Therefore, nineteen patients $(61.3 \%)$ received additional systemic chemotherapy after re-RT.

The overview of the initial RT and re-RT is shown in Tables 2 and 3. The median interval between the end of the initial RT and the start of re-RT was 15.1 months (range, 4.4 to 56.3 months). For the initial RT, the median RT dose was $60 \mathrm{~Gy}$ (range, 45 to $66 \mathrm{~Gy}$ ) and $79.2 \mathrm{~Gy}_{10}$ (range, 51.75 to $150 \mathrm{~Gy}_{10}$ ). Twenty-six patients $(83.9 \%)$ received RT via 3D-CRT, and four patients were treated with linac-based IMRT or TOMO. Concurrent chemotherapy was delivered to 19 patients (61.3\%). The median PTV was $353.15 \mathrm{~mL}$ (range, 33.3 to 679.9 $\mathrm{mL}$ ) and the median GTV was $57.4 \mathrm{~mL}$ (range, 2.83 to 211.5 $\mathrm{mL})$.

For re-RT, the median RT dose was 50 Gy (range, 35 to 65 Gy) and $68.8 \mathrm{~Gy}_{10}$ (range, 43.2 to $132 \mathrm{~Gy} 10$ ). All patients were treated using high conformal RT via IMRT or SBRT. Only three patients $(9.7 \%)$ were administered concurrent chemotherapy. Ten patients $(32.2 \%)$ showed more than 3 Gy in the RT fraction size. By a multidisciplinary team, elderly patients, patients with comorbidities, and patients with poor pulmonary function tests tend to decide to treat with radiation, especially SBRT rather than surgery. The median PTV was $51.3 \mathrm{~mL}$ (range, 13 to $299.3 \mathrm{~mL}$ ), and the median GTV was $13.45 \mathrm{~mL}$ (range, 1.4 to $124.9 \mathrm{~mL}$ ).

\section{Treatment outcomes and survival}

At the time of the last follow up, 23 patients were deceased and eight patients were alive. The median follow-up time was 17.4 months (range, 4.8 to 76.8 months). Eighteen patients $(58.1 \%)$ showed loco-regional recurrence, sixteen exhibited progression in the high-dose irradiated volume and two patients had progression of the regional nodal chain in the out-field. Eleven patients $(35.5 \%)$ showed distant metastasis after re-RT. Both local recurrence and distant metastasis after re-RT were observed in six patients. The major sites of distant metastasis were the brain and the contra-lateral lung, which occurred in four patients $(36.4 \%)$ each. Abdominal lymph node metastasis occurred in two patients $(18.2 \%)$, while hepatic metastasis occurred in one patient $(9.1 \%)$, and bone metastasis occurred in one patient $(9.1 \%)$.

The crude LC was $48.4 \%$. The 1-year and 2-year LC rates were $60.2 \%$ and $43.7 \%$, respectively. The LRFS was 15.4 months (range, 3.4 to 76.8 months). 1-Year and 2-year LRFS rates were $58.1 \%$, and $36.2 \%$, respectively (Fig. 1). The median DMFS was 29.8 months (range, 2.2 to 76.8 months). 1-Year and 2-year DMFS rates were $72.8 \%$ and $65.5 \%$, respectively. The median OS was 20.4 months (range, 4.8 to 76.8 


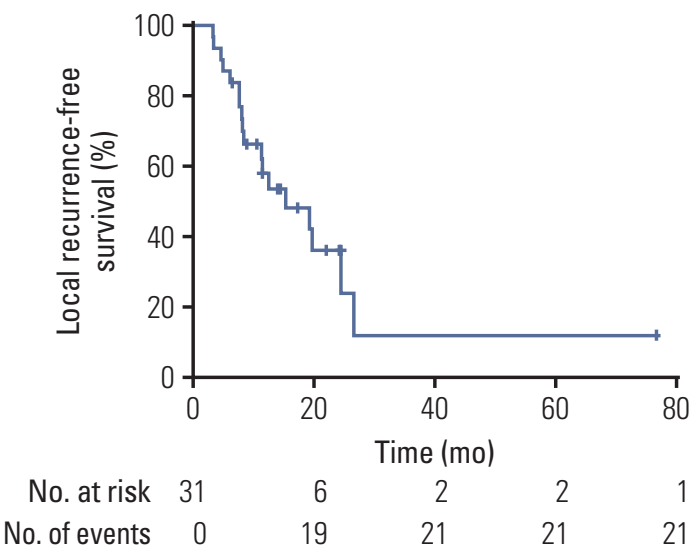

Fig. 1. Kaplan-Meier curve of local recurrence-free survival for all patients.

months). 1-Year and 2-year OS rates were 76.8\% and 39.4\%, respectively (Fig. 2).

According to univariate analysis, the cumulative $\mathrm{BED}_{10}$ $\geq 145 \mathrm{~Gy}_{10}$ and re-RT BED $10 \geq 68.7 \mathrm{~Gy}_{10}$ were significant factors for a longer OS ( $p=0.029$ and $p=0.012$, respectively). They were also associated with a longer LRFS ( $p=0.003$ and $p=0.000$, respectively). Higher cumulative $\mathrm{BED}_{10}$ and re-RT $B E D_{10}$ also typically showed trends toward longer DMFS ( $p=0.072$ and $p=0.053$, respectively). These findings demonstrate that a higher radiation dose leads to a better treatment outcome in locoregionally recurrent lung cancer. Chemotherapy after re-RT, male gender, PTV $<51.3 \mathrm{~mL}$ at re-RT, GTV $<10 \mathrm{~mL}$ at re-RT and fraction size $>3$ Gy were also significant factors for longer DMFS ( $p=0.020, p=0.034 p=0.007, p=0.024$, and $\mathrm{p}=0.006$, respectively). In those small volume tumors, highdose radiation to recurrent lung cancer resulted in a long DMFS. There were no significant differences in the OS, LRFS, and DMFS with regard to the other factors (Table 4).

\section{Toxicity}

The most frequent acute toxicity was grade 1-2 pulmonary toxicity including dyspnea, cough and pneumonitis. No grade 3-4 acute toxicities were observed. Grade 1 and 2 pulmonary toxicities were observed in 18 and 10 patients, respectively, at a median of 3.02 months (range, 0.17 to 13.9 months). The median cumulative mean lung dose (MLD) was $16.28 \mathrm{~Gy}$. There was a statistically significant difference in the cumulative MLD and acute pulmonary symptoms $(p=0.004)$. There were no statistical differences in pulmonary toxicities associated with the total $\mathrm{BED}_{10}$ dose, re-RT BED 10 dose, re-RT PTV volume, or location of the RT field, which were either central or peripheral and overlapped volume the

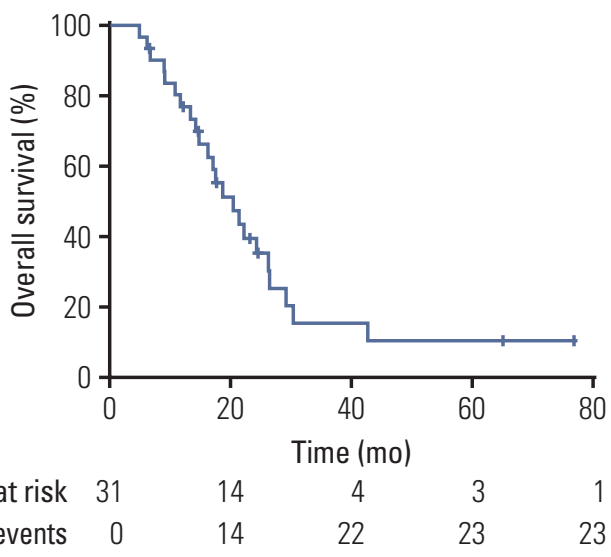

Fig. 2. Kaplan-Meier curve of overall survival for all patients.

prior RT field (Table 5).

Grade 1 and 2 esophagitis was observed in five and one patients, respectively. The median cumulative maximum esophagus dose was $124.0 \mathrm{~Gy}_{3}$, and there were no statistically significant factors that predicted esophagitis, including the cumulative maximum esophagus dose, cumulative mean esophagus dose, overlapped portion of the RT field and tumor location.

Other grade 2 toxicities, including acute or chronic chest wall pain were observed in four patients and chronic pericarditis in one patient. One patient required pericardiocentesis two years after re-RT, and he also had coronary angiography (CAG) with percutaneous coronary intervention (PCI) due to underlying cardiac artery disease. There were no grade 4 or 5 cardiac events observed.

\section{Discussion}

In this study, the outcomes of 31 lung cancer patients who received more than two courses of radiation therapy in the thorax were reviewed retrospectively. The study showed promising survival outcomes after thoracic re-RT for lung cancer with a median OS of 20.4 months and a 1-year OS of $76.8 \%$. These results compare favorably with previous studies of thoracic re-RT (14-18 months and 47\%-59\%, respectively) (Table 6) [10-15]. The statistically significant prognostic factors for longer $\mathrm{OS}$ were a cumulative $\mathrm{BED}_{10}$ dose $\geq 145$ Gy10 and re-RT BED10 dose z 68.7 Gy10. Reyngold et al. [13] and McAvoy et al. [14] also reported a significant relationship between a higher re-RT dose and longer OS. In sev- 
Table 4. Univariate analysis of clinical and dosimetric factors' effect on OS, LRFS, and DMFS

\begin{tabular}{|c|c|c|c|c|c|c|c|}
\hline \multirow[b]{2}{*}{ Variable } & \multirow[b]{2}{*}{ No. } & \multicolumn{2}{|c|}{ OS } & \multicolumn{2}{|c|}{ LRFS } & \multicolumn{2}{|c|}{ DMFS } \\
\hline & & $\begin{array}{c}\text { Median } \\
\text { OS }\end{array}$ & p-value & $\begin{array}{c}\text { Median } \\
\text { LRFS }\end{array}$ & p-value & $\begin{array}{c}\text { Median } \\
\text { DMFS }\end{array}$ & p-value \\
\hline \multicolumn{8}{|l|}{ Sex } \\
\hline Male & 27 & 22.1 & 0.085 & 19.3 & 0.933 & 64.3 & 0.034 \\
\hline Female & 4 & 16.2 & & 15.4 & & 5.6 & \\
\hline \multicolumn{8}{|l|}{ Age at re-RT (yr) } \\
\hline$\geq 60$ & 23 & 20.4 & 0.715 & 15.4 & 0.999 & 29.8 & 0.858 \\
\hline$<60$ & 8 & 17.4 & & 12.6 & & 22.0 & \\
\hline \multicolumn{8}{|l|}{ Smoking history } \\
\hline Neversmoker & 2 & 14.7 & 0.800 & 6.2 & 0.610 & - & 0.317 \\
\hline Ex-smoker/Smoker & 25 & 21.3 & & 19.3 & & 17.0 & \\
\hline \multicolumn{8}{|l|}{ Pathology } \\
\hline NSCLC & 24 & 21.3 & 0.407 & 19.3 & 0.273 & 64.3 & 0.132 \\
\hline SCLC & 7 & 17.0 & & 15.4 & & 29.8 & \\
\hline \multicolumn{8}{|l|}{$\mathrm{N}$ stage } \\
\hline N0 & 6 & 21.3 & 0.717 & - & 0.144 & - & 0.132 \\
\hline $\mathrm{N}+$ & 21 & 17.4 & & 12.6 & & 17.0 & \\
\hline \multicolumn{8}{|c|}{ Chemotherapy after re-RT } \\
\hline Yes & 19 & 21.3 & 0.717 & 11.5 & 0.137 & 29.8 & 0.020 \\
\hline No & 12 & 20.4 & & 24.6 & & - & \\
\hline \multicolumn{8}{|l|}{ Comorbidity } \\
\hline Yes & 18 & 17.0 & 0.485 & 19.3 & 0.931 & 64.3 & 0.963 \\
\hline No & 13 & 20.4 & & 15.4 & & 29.8 & \\
\hline \multicolumn{8}{|l|}{ CCRT at re-RT } \\
\hline Yes & 3 & 20.4 & 0.551 & 8.3 & 0.807 & 64.3 & 0.543 \\
\hline No & 28 & 18.6 & & 15.4 & & 29.8 & \\
\hline \multicolumn{8}{|c|}{ Interval between initial RT and re-RT } \\
\hline$<15.1$ & 15 & 18.6 & 0.601 & 12.6 & 0.170 & 64.3 & 0.724 \\
\hline$\geq 15.1$ & 16 & 20.4 & & 24.6 & & 29.8 & \\
\hline \multicolumn{8}{|l|}{ Total BED 10} \\
\hline$\geq 145$ & 12 & 22.1 & 0.029 & 26.6 & 0.003 & 64.3 & 0.072 \\
\hline$<145$ & 19 & 17 & & 11.4 & & 29.8 & \\
\hline \multicolumn{8}{|l|}{ Re-RT BED B $_{10}$} \\
\hline$\geq 68.7$ & 16 & 24.2 & 0.012 & 24.6 & 0.000 & 64.3 & 0.053 \\
\hline$<68.7$ & 15 & 16.2 & & 8.4 & & 22.0 & \\
\hline \multicolumn{8}{|l|}{ GTV at re-RT (mL) } \\
\hline$\geq 10$ & 16 & 17.4 & 0.936 & 11.5 & 0.680 & 29.8 & 0.024 \\
\hline$<10$ & 12 & 20.4 & & 15.4 & & - & \\
\hline \multicolumn{8}{|l|}{ PTV at re-RT (mL) } \\
\hline$\geq 51.3$ & 15 & 17.4 & 0.551 & 19.3 & 0.620 & 29.8 & 0.007 \\
\hline$<51.3$ & 14 & 20.4 & & 12.6 & & - & \\
\hline \multicolumn{8}{|l|}{ Fraction size (Gy) } \\
\hline$\leq 3$ & 21 & 17.0 & 0.094 & 11.5 & 0.081 & 13.3 & 0.006 \\
\hline$>3$ & 10 & 22.1 & & 24.6 & & - & \\
\hline
\end{tabular}

OS, overall survival; LRFS, loco-regional recurrence-free survival; DMFS, distant metastasis-free survival; re-RT, re-irradiation; NSCLC, non-small cell lung cancer; SCLC, small cell lung cancer; N0, no lymph node metastasis; $\mathrm{N}+$, positive for lymph node metastasis; CCRT, concurrent chemoradiation; RT, irradiation; $\mathrm{BED}_{10}$, biologically equivalent dose for $\alpha / \beta=10$; GTV, gross tumor volume; PTV, planning tumor volume. 
Table 5. Acute and chronic toxicities (CTCAE ver. 4.03)

\begin{tabular}{|c|c|c|c|c|}
\hline & Grade 1 & Grade 2 & Grade 3 & Grade 4 \\
\hline \multicolumn{5}{|l|}{ Acute toxicities } \\
\hline Esophagitis & $4(12.9)$ & 0 & 0 & 0 \\
\hline Pericarditis & $2(6.5)$ & 0 & 0 & 0 \\
\hline Pulmonary (dyspnea, cough, pneumonitis) & $7(22.6)$ & $6(19.4)$ & 0 & 0 \\
\hline Chest wall pain & $2(6.5)$ & $3(9.7)$ & 0 & 0 \\
\hline Radiation dermatitis & $2(6.5)$ & 0 & 0 & 0 \\
\hline \multicolumn{5}{|l|}{ Chronic toxicities } \\
\hline Esophagitis & $1(3.2)$ & $1(3.2)$ & 0 & 0 \\
\hline Pericarditis & $3(9.7)$ & 0 & $1(3.2)$ & 0 \\
\hline Pulmonary (dyspnea, cough, pneumonitis) & $11(35.5)$ & $4(12.9)$ & 0 & 0 \\
\hline Chest wall pain & $1(3.2)$ & $1(3.2)$ & 0 & 0 \\
\hline
\end{tabular}

Values are presented as number (\%). CTCAE, Common Terminology Criteria for Adverse Events.

eral studies, longer interval between the initial RT and re-RT $[7,16,17]$, smaller PTV [11-13,18] and PS before re-RT [7,13,19] were reported as being significant factors associated with improved OS. In this study, none of these factors showed significant differences. However, smaller GTV $<10 \mathrm{~mL}$ and PTV $<51.3 \mathrm{~mL}$ in re-RT showed trends toward longer OS, even if the trends were not statistically significant. Compared with the previous studies, the patients in this study tended to have relatively small PTV values with a median PTV value of 51.3 $\mathrm{mL}$. Almost all patients, except two who had an ECOG PS of 2, had a good PS before re-RT. The good PS and small PTV might explain the favorable OS. Based on our results and those of previous studies, to achieve better survival outcomes, patient selection might be crucial.

The median LRFS was also comparable with previous studies. In this study, the median LRFS was 15.4 months, while other studies showed an LRFS of 12.1-18 months $[11,13,14,18]$. Our definition of loco-regional recurrence was recurrence in the primary tumor and regional lymph node area. Since each study defines a loco-regional recurrence differently, such as recurrence in the high-dose irradiated volume only or in the ipsilateral lung and regional nodal area, it might be difficult to directly compare the LRFS value with those of other studies. In this study, sixteen patients showed progression or recurrence only in the high-dose irradiated volume, and only two patients showed progression in the regional lymph node. One patient had re-RT on the right paratracheal node, and then, 4.8 months later, there was progression around the descending aorta and the paraesophageal lymph node. Another patient had re-RT on the subcarinal lymph node only, and 23.1 months later, there was progression on the paratracheal station. Excluding these two patients to determine the median time to progression in the high-dose irradiated field only, which is defined as LPFS, our median LPFS was 15.4 months (range, 3.5 to 76.8 months).

The cumulative and re-RT BED 10 doses were significant factors for predicting a longer LRFS, and a high fraction size RT was marginally associated with a longer LRFS. According to De Bari et al. [20], the LC rates with stereotactic re-RT were superior to those with conventional re-RT. Sumita et al. [18] also implied that a re-RT dose $\geq 60$ Gy was a prognostic factor for longer LPFS.

Furthermore, chemotherapy after re-RT, male sex, PTV at re-RT $<51.3 \mathrm{~mL}, \mathrm{GTV}$ at re-RT $<10 \mathrm{~mL}$ and a fraction size $>3$ Gy were significantly associated with longer DMFS, and a total $\mathrm{BED}_{10}$ dose $\geq 145 \mathrm{~Gy}_{10}$, and re-RT $\mathrm{BED}_{10}$ dose $\geq 68.7$ $\mathrm{Gy}_{10}$ were associated with a marginally longer DMFS. A larger fraction size and a higher re-RT dose were predictive factors for longer LRFS, and these aggressive radiation factors improved the DMFS in recurrent lung cancer. A PTV at re-RT $<51.3 \mathrm{~mL}$ and, a GTV re-RT $<10 \mathrm{~mL}$ were significant factors for longer DMFS, which indicates that small volume tumors have a smaller cancer burden and should lead to longer DMFS. From this analysis, dose escalation should be considered in further studies to improve the outcome of re-RT treatment of small volume tumors.

Radiation-induced toxicities after re-RT remain a concern. In a pooled analysis of 14 studies, De Ruysscher et al. [8] reported an average of $7 \%$ of patients exhibiting grade 3-4 lung toxic effects and $2 \%$ grade 3 esophagitis. Grade 5 bleeding was also reported in 12 of 408 patients.

Despite concerns about radiation-induced toxicities, there were no serious acute complications. Overall, the most frequent toxicities were pulmonary toxicities, including dyspnea, cough and pneumonitis (22.6\% acute grade $1,19.4 \%$ acute grade $2,35.5 \%$ chronic grade 1 , and $12.9 \%$ chronic grade 2 ). These results showed better rates than those reported after conventional RT. Compared with previous studies, 


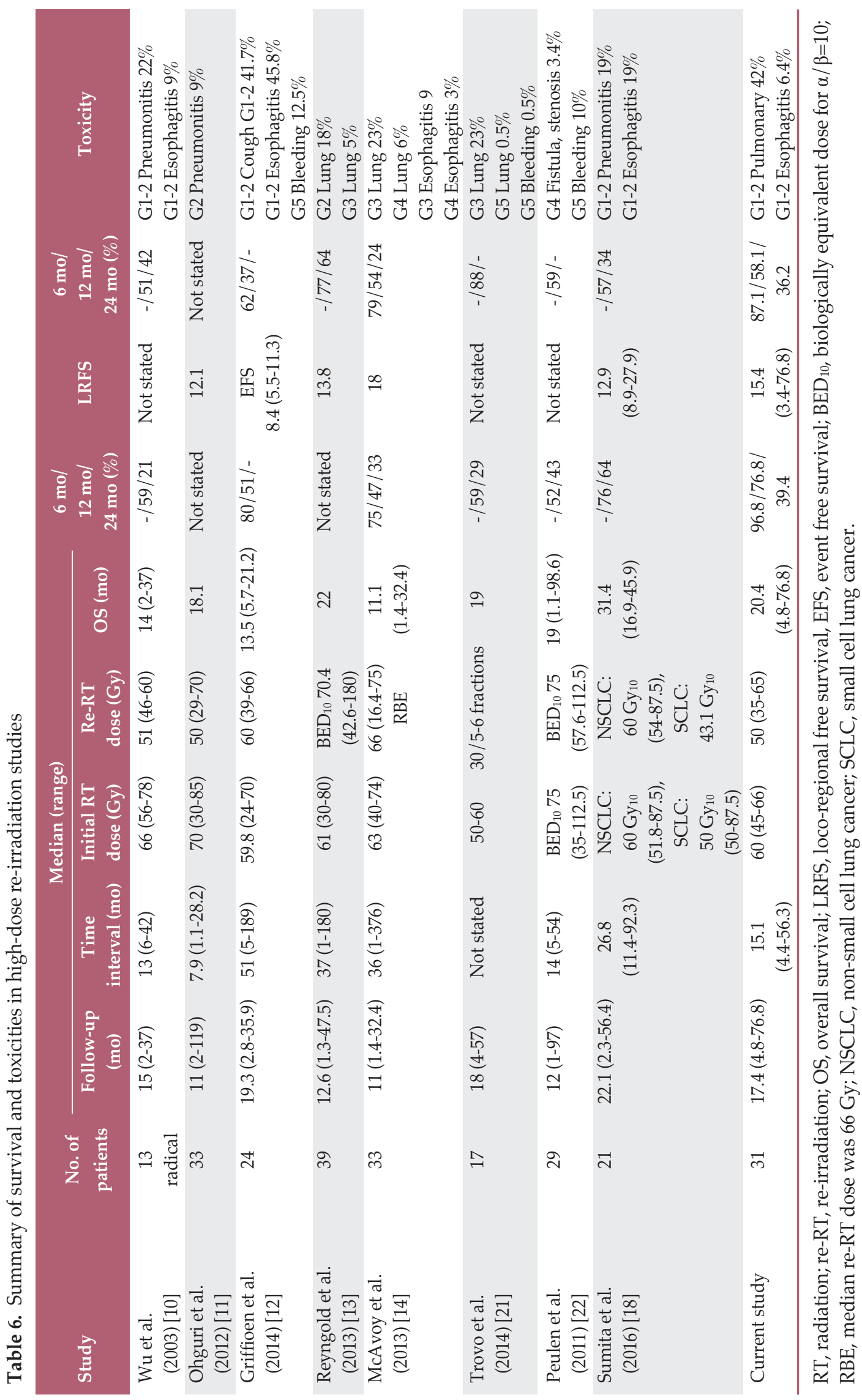



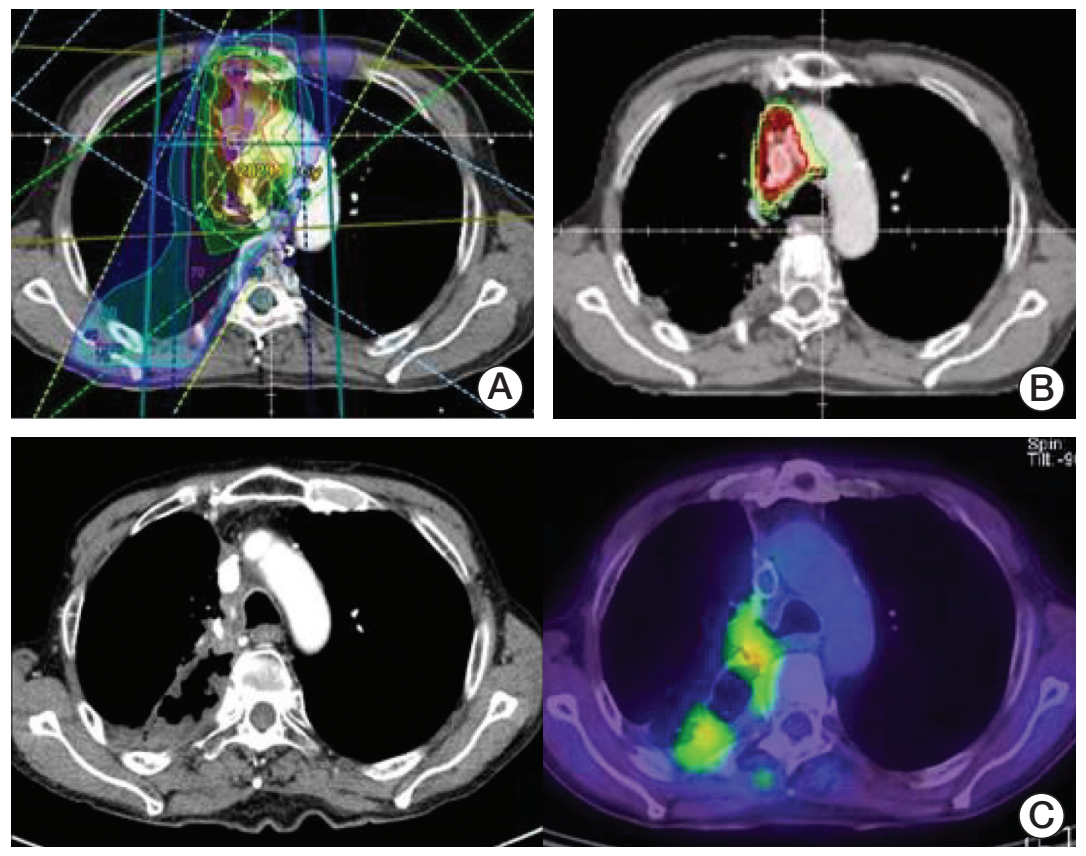

Fig. 3. The initial irradiation (RT) plan (A), re-RT plan (B), and disease progression after re-RT (C) for patient 1 who experienced bleeding in locally progressive disease after high dose re-RT.
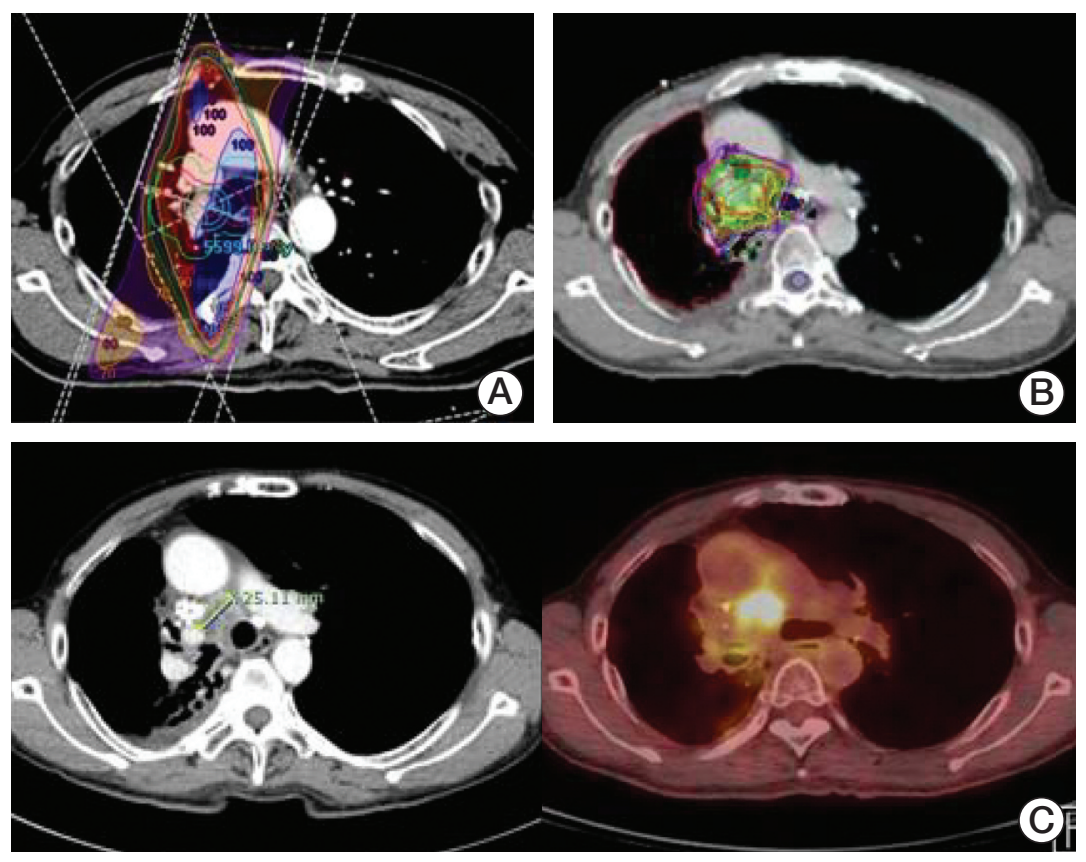

Fig. 4. The initial irradiation (RT) plan (A), re-RT plan (B), and disease progression after re-RT (C) for patient 2 who experienced bleeding in locally progressive disease after high dose re-RT. 
our results indicated similar toxicity rates (Table 6). Grade 3 pericarditis occurred in only one patient who also went through CAG with PCI due to underlying cardiac disease. This low rate of acute toxicity is probably due to the smaller RT target volumes and the high precision techniques commonly used for a course of salvage re-RT.

There were two patients of bleeding in locally progressive disease in re-RT volume. Both events were clinically diagnosed as hematemesis or hemoptysis; however, it is hard to determine the cause of the bleeding because they refused to be evaluated. Furthermore, both had taken anti-coagulants due to underlying disease. Based on clinical courses and images, it can be assumed that the causes of the bleeding are progressions of the local disease. As shown in Figs. 3 and 4, two patients had an overlapped PTV in the central and higher cumulative D max dose of esophagus and trachea. (Figs. 3 and 4).

The predictive factors for toxicity after re-RT have not been well-studied. Trovo et al. [21], Peulen et al. [22], and McAvoy et al. [15] reported that toxic effects are greater in centrally located tumors. In this study, there were no significant differences in the location of tumors. The patients in this study had small PTV values, which might have led to less damage to the normal organ in which they were located. McAvoy et al. [15] also reported that a CCRT effect may increase toxicity risk. The CCRT effect also did not show differences in this study. However, in this study, as the cumulative MLD increased, the acute pulmonary toxicity increased in a statistically significant manner.

This study has several limitations. First, the number of patients is small and the follow up period was not sufficiently long. This makes statistical analysis less accurate and may result in significant factors being missed. Because of the small number of patients, multivariate analysis was hard to be done. Statistical values with the small number of patients must be interpreted with cautions. Second, the patient' characteristics and treatment techniques were not homogeneous, containing both SBRT and IMRT, and were also not homogeneous regarding pathology, stage, and treatment course. We tried to limit the heterogeneity by selecting patients who were diagnosed as non-metastatic or who had a controlledmetastatic stage for re-RT with high-dose salvage radiation. Third, owing to the retrospective methodology, the highgrade toxicity effects might be underestimated and there might be selection-bias.

Thoracic re-RT of lung cancer has been challenged by the tolerance doses of normal tissues, such as the lung, esophagus, and trachea and there is a high possibility of acute and chronic toxicities. However, our results showed a favorable OS rate with a low rate of toxicity.

The cumulative dose and re-RT dose were significant factors in predicting both longer OS and LRFS in this study.

To improve the treatment outcome and apply a sufficiently high dose, selection of patients to receive thoracic re-RT is crucial in extending the indications of re-RT. With proper indications and application of re-RT using highly precise technique, such as IMRT and SBRT, we are able to achieve improvements in treatment outcomes with acceptable toxicity in recurrent lung cancer.

\section{Conflicts of Interest}

Conflict of interest relevant to this article was not reported.

\section{References}

1. National Cancer Information Center. Annual report of cancer statistics in Korea in 2012-2015 [Internet]. Goyang: National Cancer Information Center; 2016 [cited 2018 Mar 10]. Available from: http:// www.cancer.go.kr/.

2. Park JY, Jang SH. Epidemiology of lung cancer in Korea: recent trends. Tuberc Respir Dis. 2016;79:58-69.

3. Warde P, Payne D. Does thoracic irradiation improve survival and local control in limited-stage small-cell carcinoma of the lung? A meta-analysis. J Clin Oncol. 1992;10:890-5.

4. Oh IJ, Ahn SJ. Multidisciplinary team approach for the management of patients with locally advanced non-small cell lung cancer: searching the evidence to guide the decision. Radiat Oncol J. 2017;35:16-24.

5. Auperin A, Le Pechoux C, Rolland E, Curran WJ, Furuse K, Fournel $\mathrm{P}$, et al. Meta-analysis of concomitant versus sequen- tial radiochemotherapy in locally advanced non-small-cell lung cancer. J Clin Oncol. 2010;28:2181-90.

6. Turrisi AT 3rd, Kim K, Blum R, Sause WT, Livingston RB, Komaki R, et al. Twice-daily compared with once-daily thoracic radiotherapy in limited small-cell lung cancer treated concurrently with cisplatin and etoposide. N Engl J Med. 1999;340:265-71.

7. Jeremic B, Videtic GM. Chest reirradiation with external beam radiotherapy for locally recurrent non-small-cell lung cancer: a review. Int J Radiat Oncol Biol Phys. 2011;80:969-77.

8. De Ruysscher D, Faivre-Finn C, Le Pechoux C, Peeters S, Belderbos J. High-dose re-irradiation following radical radiotherapy for non-small-cell lung cancer. Lancet Oncol. 2014; 15:e620-4.

9. Vassil AD, Videtic GM, Stephans KL. Re-irradiation in lung 
cancer. J Radiat Oncol. 2015;4:129-39.

10. Wu KL, Jiang GL, Qian H, Wang LJ, Yang HJ, Fu XL, et al. Three-dimensional conformal radiotherapy for locoregionally recurrent lung carcinoma after external beam irradiation: a prospective phase I-II clinical trial. Int J Radiat Oncol Biol Phys. 2003;57:1345-50.

11. Ohguri T, Imada H, Yahara K, Moon SD, Yamaguchi S, Yatera $\mathrm{K}$, et al. Re-irradiation plus regional hyperthermia for recurrent non-small cell lung cancer: a potential modality for inducing long-term survival in selected patients. Lung Cancer. 2012;77:140-5.

12. Griffioen GH, Dahele M, de Haan PF, van de Ven PM, Slotman BJ, Senan S. High-dose, conventionally fractionated thoracic reirradiation for lung tumors. Lung Cancer. 2014;83:356-62.

13. Reyngold M, Wu AJ, McLane A, Zhang Z, Hsu M, Stein NF, et al. Toxicity and outcomes of thoracic re-irradiation using stereotactic body radiation therapy (SBRT). Radiat Oncol. 2013;8:99.

14. McAvoy SA, Ciura KT, Rineer JM, Allen PK, Liao Z, Chang JY, et al. Feasibility of proton beam therapy for reirradiation of locoregionally recurrent non-small cell lung cancer. Radiother Oncol. 2013;109:38-44.

15. McAvoy S, Ciura K, Wei C, Rineer J, Liao Z, Chang JY, et al. Definitive reirradiation for locoregionally recurrent non-small cell lung cancer with proton beam therapy or intensity modulated radiation therapy: predictors of high-grade toxicity and survival outcomes. Int J Radiat Oncol Biol Phys. 2014;90:81927.
16. Tada T, Fukuda H, Matsui K, Hirashima T, Hosono M, Takada $\mathrm{Y}$, et al. Non-small-cell lung cancer: reirradiation for locoregional relapse previously treated with radiation therapy. Int J Clin Oncol. 2005;10:247-50.

17. Trakul N, Harris JP, Le QT, Hara WY, Maxim PG, Loo BW Jr, et al. Stereotactic ablative radiotherapy for reirradiation of locally recurrent lung tumors. J Thorac Oncol. 2012;7:1462-5.

18. Sumita K, Harada H, Asakura H, Ogawa H, Onoe T, Murayama $S$, et al. Re-irradiation for locoregionally recurrent tumors of the thorax: a single-institution, retrospective study. Radiat Oncol. 2016;11:104.

19. Aktan M, Kanyilmaz G, Koc M, Aras S. Thoracic re-irradiation for locally recurrent lung cancer. Asian Pac J Cancer Prev. 2016;17:5041-5.

20. De Bari B, Filippi AR, Mazzola R, Bonomo P, Trovo M, Livi L, et al. Available evidence on re-irradiation with stereotactic ablative radiotherapy following high-dose previous thoracic radiotherapy for lung malignancies. Cancer Treat Rev. 2015; 41:511-8.

21. Trovo M, Minatel E, Durofil E, Polesel J, Avanzo M, Baresic T, et al. Stereotactic body radiation therapy for re-irradiation of persistent or recurrent non-small cell lung cancer. Int J Radiat Oncol Biol Phys. 2014;88:1114-9.

22. Peulen H, Karlsson K, Lindberg K, Tullgren O, Baumann P, Lax I, et al. Toxicity after reirradiation of pulmonary tumours with stereotactic body radiotherapy. Radiother Oncol. 2011; 101:260-6. 